\title{
Imaging Macromolecules and Viruses in a Hydrated State Using a Field Emission Scanning Electron Microscope (FESEM)
}

\author{
C.A. Ackerley ${ }^{1}$, C Nielsen ${ }^{2}$ and G. Rodek $^{3}$ \\ ${ }^{1}$ Division of Pathology, Hospital for Sick Children, Toronto, Canada \\ 2 JEOL USA, Peabody, MA, USA \\ ${ }^{3}$ SPI Supplies, West Chester, PA, USA
}

A capsule capable of retaining liquid at one atmosphere with an electron lucent partition membrane was developed for the observation of cells and other materials in a hydrated state in the scanning electron microscope (SEM) was developed in the early 2000s [1]. Cells can be grown on the internal face of the electron lucent partition membrane and processed using a number of protocols including conventional electron microscopy fixation and staining procedures, cytochemistry and immunogold immunocytochemistry. The samples are then immersed in a media which dissipates electrons. The capsule is sealed and the cells imaged using backscatter electron (BE) imaging. More recently, we have significantly modified the capsule and have replaced the polymer partition window with a $20 \mathrm{~nm}$ thick film of silicon oxide. Using a FESEM and a phosphor coated BE detector we have been able to obtain time lapse serial images of immunogold labelled live cells for up to 5001.8 second scans at approximately 30 second intervals. Cells demonstrated at least $80 \%$ viability using an absorbed current of 10pA[2].

In a previous experiment, we had evaluated the sensitivity of three BE electron detectors and an in lens Wien based filter using NIH Image J and a SMART macro [3]. Although the BE detector with the phosphor scintillator was the most electron dose sensitive, resolution was impaired by its position in the column. Specimens were limited to a working distance of 8mm. Using the in lens Wien filter,the sample can be used at very short working increasing the resolution of the images. An added bonus to using this imaging mode is that the majority of the atomic contrast is generated from the surface of the object and electrons originating from within the sample contribute very little to the final product.

The first hurdle to overcome in imaging hydrated small organic objects was developing a protocol for adhering the specimens to the inner face of the partition window. A capsule was redesigned where several $\mu \mathrm{l}$ of the solution containing either the macromolecules or the viruses was administered to the inner surface of the partition membrane. The vessel which contains the partition membrane was threaded and contained a relief hole to minimize pressure to the fragile partition membrane. The backing plate was then screwed into the vessel until no further fluid was coming out of the relief hole. The relief hole was then sealed with a small drop of cyanoacrylate glue. This results in a very thin film of solution containing the object to be studied in intimate contact with the partition membrane.

We are currently using either $0.01 \%$ uranyl acetate, $0.02 \%$ ammonium molybdate or no contrasting additive in the specimen suspension. We have limited our studies to adenovirus, microtubules and glycogen. All three of the specimen types are easily imaged at near TEM resolutions when the specimen is suspended in solution with a contrasting additive (fig.1,2). 
Unstained samples were very difficult to see however with some further image processing the structures were quite detectable.

Chamber design is now being revisited. The most successful modification to date has been the inclusion of another silicon oxide membrane into the blanking plate eliminating the majority of the backscatter electrons contributed from the more robust standard backing plate. Using this design the success rate of eliminating the excess specimen fluid is quite difficult as either the top or bottom membranes are easily broken during the removal of the excess specimen fluid. When we have been successful at this, unstained specimen visibility increases several fold. Like our previous studies with live cells it is hoped that using this technique we will be able to serial image molecular interactions in these chambers.

\section{References:}

[1] S. Thiberge et al., Proc Natl Acad Sci USA 10(10) (2004) 3346.

[2] C.A. Ackerley et al., Microsc and Microanal12(2) (2006) 428CD.

[3] D.C. Joy, J Microsc (1) (2004) 24.

[4] The authors acknowledge funding from the Hammond Foundation.
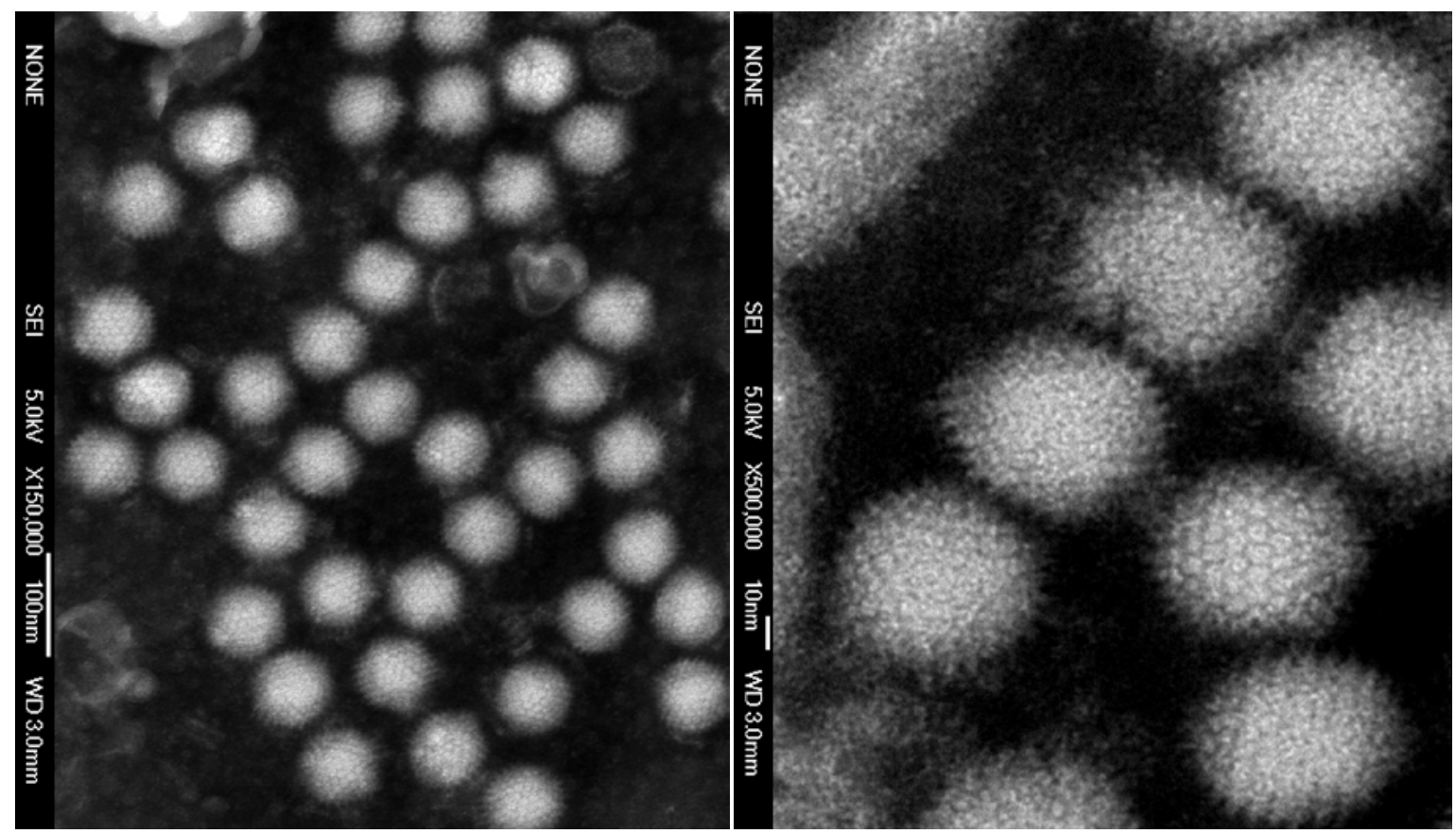

Fig.1 In lens Wien filtered image of hydrated Fig.2 In lens Wien filtered image of hydrated adenovirus in a solution containing $0.01 \%$ adenovirus in a solution containing $0.02 \%$ uranyl acetate. Contrast has been reversed. ammonium molybdate. Contrast has been reversed. 